\title{
Research of Non-Standard Disc Spring for Anti-Stall Tool
}

\author{
Jialin Tian $^{1,2}$, Xudong Cai ${ }^{1}$, Tangjia Zhang ${ }^{1}$ \\ ${ }^{1}$ School of Mechatronic Engineering, Southwest Petroleum University, Chengdu, 610500, China \\ ${ }^{2}$ School of Mechanical Engineering, Southwest Jiaotong University, Chengdu, 610031, China
}

\begin{abstract}
To solve the phenomenon of sticking of tool that occurred in the process of drilling a ultra-deep well and extended-reach well, an anti-stall tool is designed. During the axial contraction and extension of spiral spline shaft of the anti-stall tool, The disc spring system plays the role of storage and release energy. For further understanding, the working performance of the disc spring, combining with the working parameters of the anti-stall tool, the load-displacement characteristics, stiffness characteristics and stress characteristics of the disc spring are analyzed. The analysis results show that the mechanical properties of the disc spring can meet the requirements of the anti-stall tool, which providing a reference and guiding significance for optimizing the working characteristics of the anti-stall tool.
\end{abstract}

Keywords: Anti-stall tool; Disc spring; Load displacement characteristics; Stiffness characteristics; Stress characteristics

\section{Introduction}

Disc Spring is a powerful storage and releases mechanical component, which is widely used in mechanical products. Because disc spring is possession of buffer, damping capacity and great stiffness, it is mainly used in heavy machinery fields ${ }^{[1-4]}$. With the increasing demands of oil and natural gas, the exploitation of oil and gas is in the shallow stratum, and gradually turned to complex formation, such as deep well, ultra-deep well, and extended reach well. To settle card drilling, stick-slip during drilling in ultra-deep wells, extended reach well, various kinds of downhole tools have been proposed, which have a significant effect in practical application, and the drilling efficiency is significantly improved ${ }^{[5-6]}$. In the process of drilling, the size of the drilling tool is strictly restricted due to the change of the scale of the hole and the hole trajectory ${ }^{[7-8]}$. By stiffness property, small deformation bearing large load and required little axial space of disc spring, a new type of anti pipe-sticking, reducing the slimy anti- stall drilling tool is designed, which mainly contains two parts, top device, and bottom device, the top device is actually disc spring combination, the lower device is multi heads spiral spline pairs. In the anti-stall tool, disc spring plays the role of absorbing the axial vibration, storing and releasing energy and controlling the axial movement.

To better understand and grasp the characteristics of the disc spring, a variety of methods have been used in the study of disc spring in the related field. Combining with the actual structure and working mode of the disc spring, $\mathrm{Yi}$ Xianzhong drew the conclusion of an analytical solution for the new design of the disc spring model and studied the characteristics of stiffness, deformation energy, and self-vibration frequency of disc spring ${ }^{[9]}$. Wang Xiaobo utilized experiment test data, finite element software LS-DYNA and ANSYS to have a numerical calculation and analysis of fundamental mechanical properties of disc spring combination, drew the harmonic response of the disc spring, found out the dangerous points of easily damaged ${ }^{[10]}$. S. Ozaki considered static and dynamic loading property of disc spring combination, calculated cone friction coefficient equivalent value of disc spring ${ }^{[11]}$. Hashiguchi $\mathrm{K}$ and Zheng Lijuan combined with the actual working characteristics of disc spring, the correction formula of the disc spring load considering the influence of friction is given. The results of the theoretical analysis are verified by numerical simulation $^{[12]}$.

In the foundation of existing research, this paper establishes a two stack of eighty pairs of composite disc spring combination model which is utilized for top device of anti-stall tool, analyzes mechanical properties of the model by the theoretical calculation method, the change of the disc spring combination under the load is obtained, provides a theoretical reference for the design and optimization of the anti-stall tool.

\section{Model Building and Theoretical Analysis}


International Journal of Science and Research (IJSR)

ISSN (Online): 2319-7064

Index Copernicus Value (2016): 79.57 | Impact Factor (2015): 6.391

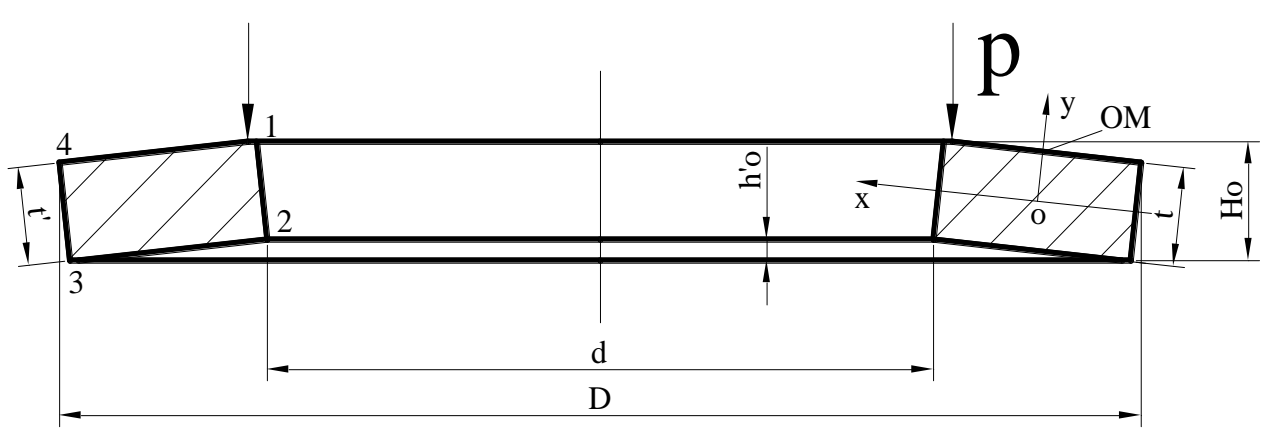

Figure 1: Structural diagram of non-standard disc springs with support surface

Fig.1. Shows a structural diagram of non-standard disc spring with support surface used in the anti-stall tool. The main dimensions of the disc spring as follows: Outside diameter $D=125 \mathrm{~mm}$, Inside diameter $d=77 \mathrm{~mm}$, Cone height $h_{0}^{\prime}=2.37 \mathrm{~mm}$, The free height of the single disc spring $H_{0}=13.42 \mathrm{~mm}$, The thickness of the disc spring with support surface $t^{\prime}=11.11 \mathrm{~mm}$, and The thickness of the disc spring without support surface $t=11.22 \mathrm{~mm}$. $\delta$ is used to show the compression deformation of the disc spring under the load of $p$, the relationship between the two is as follows ${ }^{[13]}$ :

$$
p=\frac{4 E t^{\prime 4}}{\left(1-\mu^{2}\right) K_{1} D^{2}} K_{4}^{2} \frac{\delta}{t^{\prime}}\left[K_{4}^{2}\left(\frac{h_{0}^{\prime}}{t^{\prime}}-\frac{\delta}{t^{\prime}}\right)\left(\frac{h_{0}^{\prime}}{t^{\prime}}-\frac{\delta}{2 t^{\prime}}\right)+1\right]
$$

Where $\mathrm{E}$ is elastic modulus, $\mathrm{MPa} ; \mu$ is Poisson ratio;

$K_{1} 、 K_{4}$ is calculating coefficient.

$$
p_{c}=\frac{4 E}{1-\mu^{2}} \times \frac{t^{\prime 3} h_{0}^{\prime}}{K_{1} D^{2}} K_{4}^{2}
$$

Where $p_{c}$ is a load of disc spring flattened out

$$
K_{1}=\frac{1}{\pi} \frac{[(C-1) / C]^{2}}{(C+1) /(C-1)-2 / \ln C}
$$

Where $\mathrm{C}$ is the ratio of inner diameter and the outer diameter of disc spring.

$$
\begin{gathered}
C=\frac{D}{d} \\
K_{4}=\sqrt{-C_{1} / 2+\sqrt{\left(C_{1} / 2\right)^{2}+C_{2}}} \\
C_{1}=\frac{\left(t^{\prime} / t\right)^{2}}{\left(\frac{H_{0}}{4 t}-\frac{t}{t}+\frac{3}{4}\right)\left(\frac{5 H_{0}}{8 t}-\frac{t}{t}+\frac{3}{8}\right)} \\
C_{2}=\frac{C_{1}}{\left(t^{\prime} / t\right)^{3}}\left[\frac{5}{32}\left(\frac{H_{0}}{t}-1\right)^{2}+1\right]
\end{gathered}
$$

In the anti-stall tool, the combined disc spring is installed at the top of the tool, and the movement form of the tool is pre-compressed, compressed and restored. Before the antistall tools installed and placed in the well, it is necessary to impose some loads on the combination disc spring, make the combination disc spring generate a certain preload, which loads on the bottom device of the spiral spline pair to set a preset torque. In the working process of the anti-stall tool, when the reverse torque of the drill bit is bigger than the preset torque, the spiral spline shaft would move upward and compress the combined disc spring, which makes the disc spring system store energy ; As the reverse torque of drill bit reducing, the disc spring system would restore, release the energy to drive the long spiral outer spline shaft gradually downward, so that the drill bit continues smoothly drilling. To study the mechanical behavior of the disc spring system, a simplified model is established, which shows Fig. 2.

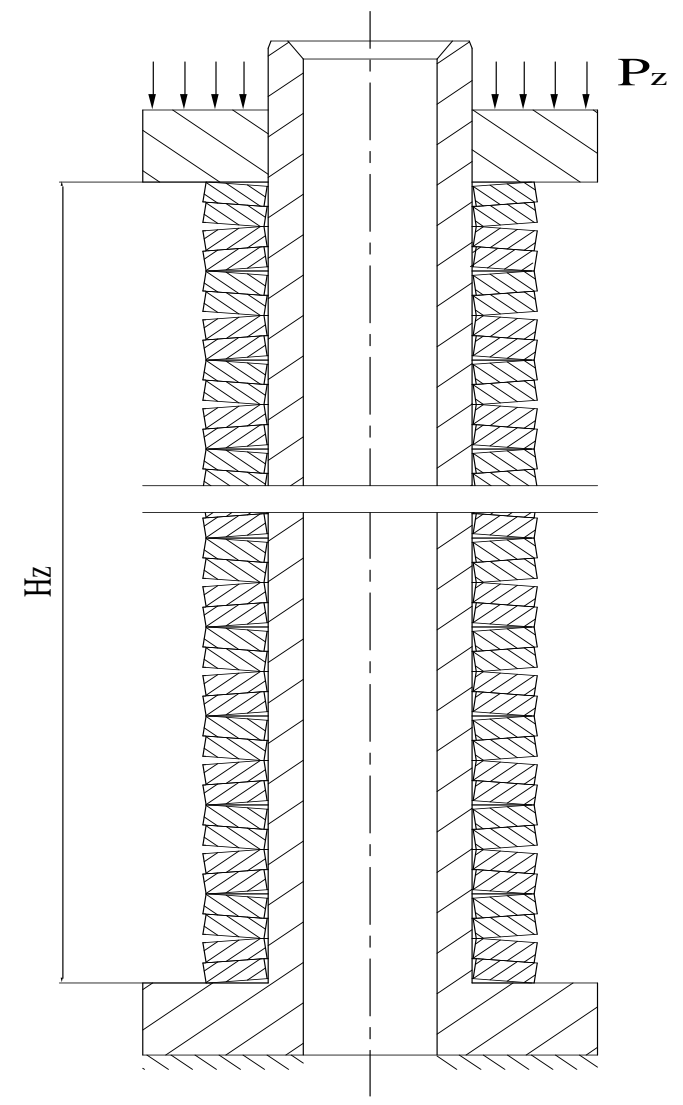

Figure 2: The design diagram of disc spring combinations

In Figure 2, to meet the requirements of the axial contraction

Volume 6 Issue 12, December 2017 


\section{International Journal of Science and Research (IJSR) \\ ISSN (Online): 2319-7064 \\ Index Copernicus Value (2016): 79.57 | Impact Factor (2015): 6.391}

and extension and the torque value in the working process, the disc spring system is adopted in the 160 piece combination ( 2 pieces of 80 overlapping groups). The relationship between the deformation of the disc spring system under external load and the external force as follows:

$$
p_{z}=\frac{n p}{1 \mp f_{M}(n-1)}
$$

Where $p_{z}$ is the load of the disc spring system; $\mathrm{P}$ is the load of the single disc spring without friction, $\mathrm{KN}$; $\mathrm{n}$ is the number of laminated disc springs; $f_{M}$ is the coefficient of friction between the conical disc springs.

In Eq.(8), when increasing load, choose -, when reducing load, choose + .

In the disc spring system, the total deformation and free height of the combined disc spring are presented by the following relationship:

$$
\delta_{z}=i \delta
$$

Where $\delta_{z}$ is the deformation of the disc spring system; $i$ is the number of the couple disc springs; $\delta$ is a single disc spring deformation.

$$
H_{z}=i\left[H_{0}+(n-1) t^{\prime}\right]
$$

Where: $H_{z}$ is the free height of the disc spring system

\section{Calculation and analysis of mechanical properties}

\subsection{Analysis of load-displacement characteristics}

The material for the non-standard disc spring used in the anti-stall tool is 50CrVA, if $D / t^{\prime}=11.25$ $h_{0}^{\prime} / t^{\prime}=0.21$, this disc spring belongs to the A series, Material modulus of elasticity $E=206000 \mathrm{MPa}$, Poisson ratio $\mu=0.3$.

According to the known parameter value, from the formula (1) to formula (7), the relationship between the single chip with the support surface of the non-standard disk spring deformation $\delta$ and loads $p$ can be obtained as shown in Fig.3.

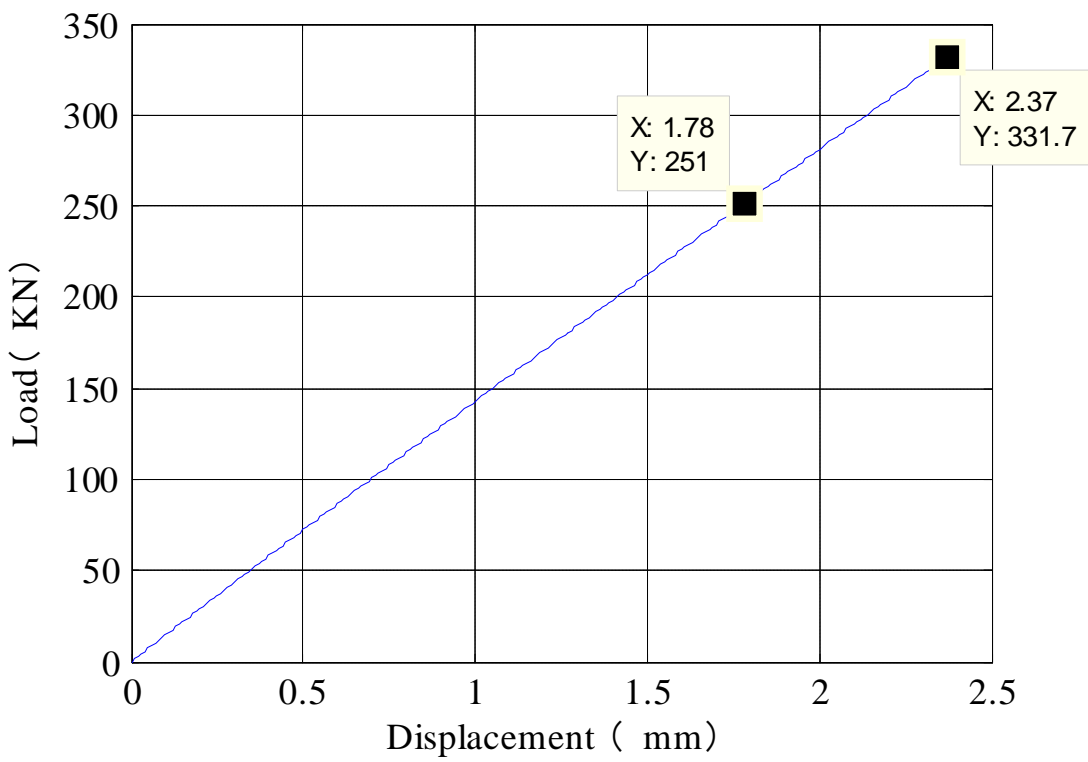

Figure 3: Loading-displacement curve of single disc spring

The relationship between the displacement of a single plate with a support plane and a load of non-standard disc spring presented in Fig. 3. Under the function of load, the deformation displacement and load of the single plate, spring has a linear relationship with load. In which, the point $(1.78,251)$ is the load corresponding to the maximum deformation amount of $\delta=0.75 h^{\prime}$ in the working process of the disc spring, the point $(2.37,331.7)$ is the maximum load of disc spring flattened corresponding to the maximum load $p_{c}$.

In order to actually understand the relationship between the deformation of disc spring and the load used in the anti-stall tool, the friction coefficient of non-standard disc spring cones with the supporting surface $f_{M}=0.03$, combined

\section{Volume 6 Issue 12, December 2017}




\section{International Journal of Science and Research (IJSR) \\ ISSN (Online): 2319-7064 \\ Index Copernicus Value (2016): 79.57 | Impact Factor (2015): 6.391}

with the formula (8), the relationship between the

drawing as shown in Figure 4.

deformation and the load of the disc spring system of

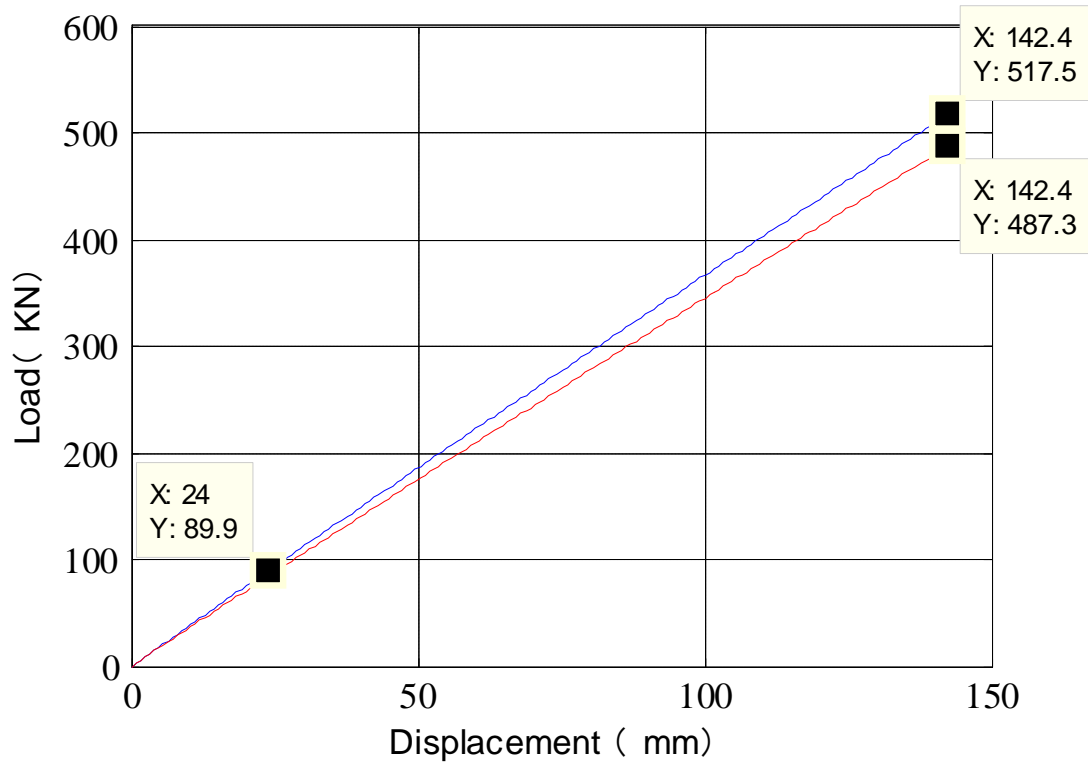

Figure 4: Loading-displacement curve of disc spring system

From Figure 4, it can be found that in considering the disc spring cone friction coefficient the curves of force and displacement of the disc spring system shows linear relationship when increasing load or reducing load, at the same time, if the changes of the displacement phase are the same, the increasing load force is greater than the corresponding reducing load force. As is shown in the Fig.4. When the maximum deformation of the disc spring system corresponding to the maximum load point $(142.2,517.5)$, the maximum deformation $\delta_{\max }=142.2 \mathrm{~mm}$ meets the maximum stroke of the anti-stall tool. In the installation of disc spring system, it is necessary to have a pre-compression for the disc spring to set the preset torque for the spiral spline pair, the point $(24,89.9)$ in the Fig.4.shows the relationship between pre-compression and loading load of the disc spring system.

\subsection{Analysis of static stiffness characteristic}

Static stiffness analysis of single disc spring:

$$
k=\frac{4 E}{1-\mu^{2}} \times \frac{t^{\prime 3}}{K_{1} D^{2}} K_{4}^{2}\left\{K _ { 4 } ^ { 2 } \left[\left(\left(\frac{h_{0}^{\prime}}{t^{\prime}}\right)^{2}-3 \frac{h_{0}^{\prime}}{t^{\prime}} \frac{\delta}{t^{\prime}}+\frac{3}{2}\left(\frac{\delta}{t^{\prime}}\right)^{2}\right)+1\right.\right.
$$

Static stiffness analysis of disc spring system:

$$
k_{z}=p_{z} / \delta_{z}
$$

Where: $k_{z}$ is the static stiffness of the disc spring system.

The stiffness of the disc spring is the load required to produce the unit displacement. Combining with the Eq. (11) and the Eq. (12) to analyze the static stiffness of the single disc spring and the disc spring system in the anti-stall tool. 


\title{
International Journal of Science and Research (IJSR) \\ ISSN (Online): 2319-7064
}

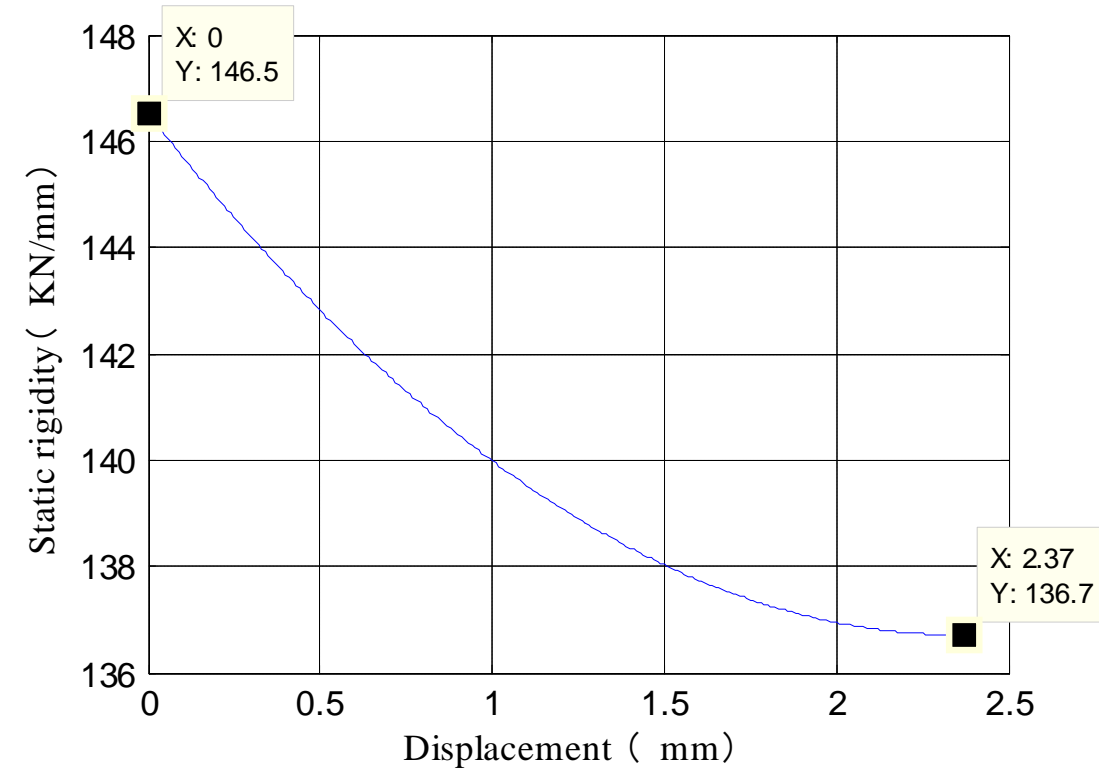

Figure 5: Rigidity-displacement curve of single disc spring

According to Fig.5.the rigidity-displacement curve of the single disc, spring has a nonlinear relationship, with the increase of the deformation of the disc spring, the static stiffness decreases gradually, and the amplitude of the reduction decreases gradually. In the whole process of deformation, when the disc spring is never flatted, Never in the spring to flatten the deformation of whole deformation process, the variation range of the static stiffness is low, decreasing from $146.4 \mathrm{KN} / \mathrm{mm}$ to $136.7 \mathrm{KN} / \mathrm{mm}$.

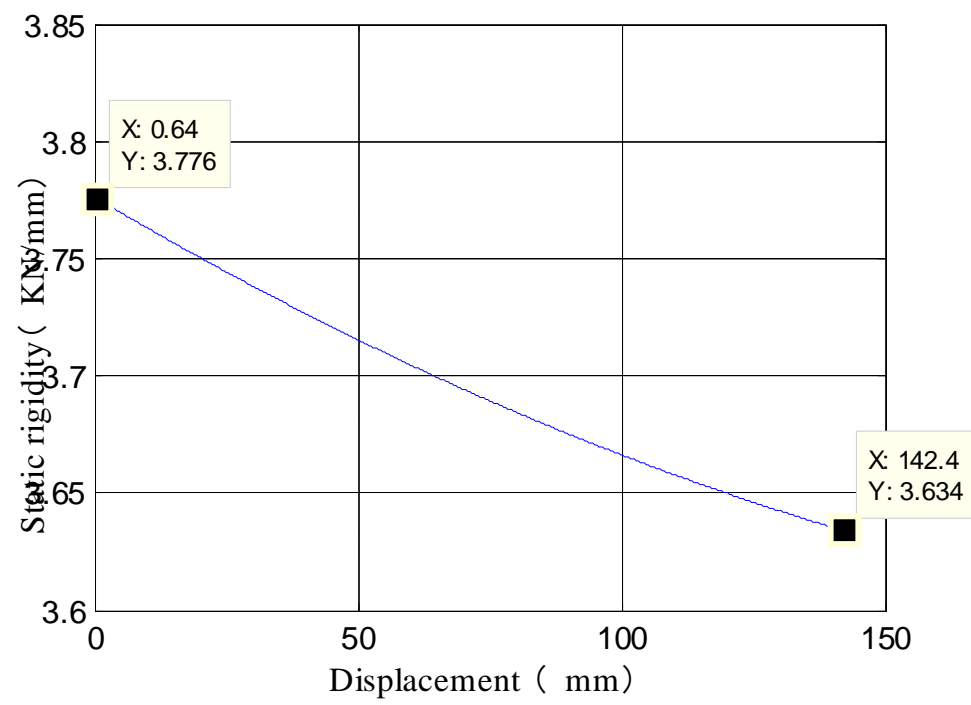

Figure 6: Rigidity-displacement curve of disc spring system

According to Fig.6. The rigidity is closely related to the combination form of the disc spring. Compared with the stiffness of the disc spring system ( 2 pieces of 80 overlapping groups) in figure 6 and the stiffness of single disc spring, when the load is increased about two times, the stiffness decreased obviously.

\subsection{Analysis of Stress characteristics}

To analyze the stress and deformation of the disc spring under load is the important theoretical basis of the design of the disc spring. The material of the disc spring has a high elastic limit, yield limit, impact resistance and plastic deformation, The material used for the disc spring in the anti-stall tool is 50CrVA. To determine the normal operation of the anti-stall tool, the stress of point 1,2, 3, 4 and OM in Figure 1 are calculated according to the following formula:

$$
\sigma_{O M}=-\frac{4 E}{1-\mu^{2}} \square \frac{t^{\prime 2}}{K_{1} D^{2}} \square K_{4} \frac{\delta}{t^{\prime}} \frac{3}{\pi}
$$

$$
\sigma_{1}=-\frac{4 E}{1-\mu^{2}} \times \frac{t^{\prime 2}}{K_{1} D^{2}} K_{4} \frac{\delta}{t^{\prime}}\left[K_{4} K_{2}\left(\frac{h_{0}^{\prime}}{t^{\prime}}-\frac{\delta}{2 t^{\prime}}\right)+K_{3}\right]
$$

Volume 6 Issue 12, December 2017

\author{
www.ijsr.net
}

Licensed Under Creative Commons Attribution CC BY 


$$
\begin{array}{r}
\sigma_{2}=-\frac{4 E}{1-\mu^{2}} \times \frac{t^{\prime 2}}{K_{1} D^{2}} K_{4} \frac{\delta}{t^{\prime}}\left[K_{4} K_{2}\left(\frac{h_{0}^{\prime}}{t^{\prime}}-\frac{\delta}{2 t^{\prime}}\right)-K_{3}\right] \\
\sigma_{3}=-\frac{4 E}{1-\mu^{2}} \times \frac{t^{\prime 2}}{K_{1} D^{2}} K_{4} \frac{1}{C} \frac{\delta}{t^{\prime}}\left[K_{4}\left(K_{2}-2 K_{3}\right)\left(\frac{h_{0}^{\prime}}{t^{\prime}}-\frac{\delta}{2 t^{\prime}}\right)-K_{3}\right] \\
\sigma_{4}=-\frac{4 E}{1-\mu^{2}} \times \frac{t^{\prime 2}}{K_{1} D^{2}} K_{4} \frac{1}{C} \frac{\delta}{t^{\prime}}\left[K_{4}\left(K_{2}-2 K_{3}\right)\left(\frac{h_{0}^{\prime}}{t^{\prime}}-\frac{\delta}{2 t^{\prime}}\right)+K_{3}\right]
\end{array}
$$

Where:

$$
\begin{gathered}
K_{2}=\frac{6}{\pi} \frac{(C-1) / \ln C-1}{\ln C} \\
K_{3}=\frac{3}{\pi} \frac{C-1}{\ln C}
\end{gathered}
$$

During working process, the axial moving distance designed in $96.4 \mathrm{~mm}$ in the anti-stall tool, the maximum load of the disc spring system is $438 \mathrm{KN}$. Because the disc spring system is two pieces of composite 80 groups of the combination form, by the formula (8), the maximum load of a single disc spring is $p=212 \mathrm{KN}$. According to $p / \mathrm{p}_{\mathrm{c}}=0.64$, $K_{4} \frac{h_{0}^{\prime}}{t^{\prime}}=0.22$, it can be drawn from the mechanical design manual in the single disc spring characteristic curve that $\delta / h_{0}^{\prime}=0.62$, at this time, the single disc spring deformation $\delta=1.47 \mathrm{~mm}$.

Under the static load, the disc spring ensures the stability of the free height $H_{0}$ by testing the stress of the point OM,

When the disc spring is pressed, $\sigma_{O M}$ should be close to (less than) the yield limit of the disc spring $\sigma_{\mathrm{s}}$. According to Fig.1.Substituting $\delta=1.47 \mathrm{~mm}$ into Eq.(13) gives the stress value $\sigma_{O M}=-1570 \mathrm{MPa}$ of the point $\mathrm{OM}$ of the disc spring being pressed. The numerical value satisfies the range of the 50CrVA yield limit.

Because the anti-stall tool can be axial contraction and extension, the disc spring system is subjected to variable load during the operation process. For the fatigue limit of 50CrVA which is used in non-standard disc spring with support surface, according to life requirement, calculating the stress intensity of key point during the working process of disc spring which is applied cyclic loading from $p_{1}=100 \mathrm{KN}$ to $p_{2}=240 \mathrm{KN}$.

To determine the key parts of fatigue of the disc spring, by $C=1.62, K_{4} \frac{h_{0}^{\prime}}{t^{\prime}}=0.22$, according to the key parts of the disc spring fatigue failure in the mechanical design manual, it can be assigned that the essential part of the fatigue failure of the disc spring is at the points in Fig.1.

According to the Eq. (8) Moreover, the characteristic curve of a single chip in mechanical design manual, It can be concluded that applying the load $p_{1}, p_{2}$ respectively on the disc spring, the deformation is respectively $\delta_{1}=0.33 \mathrm{~mm}$, $\delta_{2}=0.8 \mathrm{~mm} \quad \cdot \quad$ Substituting $\quad \delta_{1}=0.33 \mathrm{~mm}$ $\delta_{2}=0.8 \mathrm{~mm}$ into Eq.(15) gives:

$$
\left\{\begin{array}{l}
\delta_{1}=0.33 \mathrm{~mm}, \quad \sigma_{2}=367 \mathrm{MPa}=\sigma_{\text {min }} \\
\delta_{2}=0.8 \mathrm{~mm}, \quad \sigma_{2}=918 \mathrm{MPa}=\sigma_{\text {max }}
\end{array}\right.
$$

Computational stress pair of single disc spring:

$$
\sigma_{\mathrm{a}}=\sigma_{\text {max }}-\sigma_{\text {min }}=551 \mathrm{MPa}
$$

When $\sigma_{\text {rmin }}=367 \mathrm{MPa}$, according to the limit stress curve of the disc spring in the mechanical design manual,

\section{Volume 6 Issue 12, December 2017}




\section{International Journal of Science and Research (IJSR) \\ ISSN (Online): 2319-7064}

Index Copernicus Value (2016): 79.57 | Impact Factor (2015): 6.391

concluding that if $\mathrm{N}=10^{\wedge} 5$, the fatigue strength limit is

$\sigma_{\text {rmax }}=940 \mathrm{MPa}$, and the corresponding fatigue strength

stress pair:

$$
\sigma_{\mathrm{ra}}=\sigma_{\mathrm{r} \max }-\sigma_{\mathrm{r} \min }=573 \mathrm{MPa}
$$

As is shown in Eq.(21) and Eq.(22), $\quad \sigma_{\text {ra }}>\sigma_{\text {a }}$, so the disc spring can work continuously.

\section{Conclusion}

In order to satisfy the working characteristic of the anti-stall tool, the non-standard disc spring is designed. Through the analysis of the deformation and displacement of the single plate spring and the combined disc spring system under the load, it is obtained that the maximum weight of the single plate spring and disc spring system. The load and displacement change of the disc spring system meet the requirement of the modification range and load bearing requirement of the axial contraction and extension of the anti-stall tool.

Through the analysis of the static stiffness of single disc spring and disc spring system, finding that with the increase of the deformation and displacement of the disc spring, the stiffness of the disc spring decreases gradually, but the whole rigidity decreased small. Through the analysis of the stress of the disc spring, finding that the disc spring system under the maximum load $438 \mathrm{KN}$ satisfies the requirement of the yield limit of the disc spring material 50CrVA, under cyclic variable load $100 \mathrm{KN}-240 \mathrm{KN}$, the tool can meet the requirement of continuous work. These studies provide reference and guiding significance for the better use of the disc spring, playing job performance of anti-stall tools.

\section{References}

[1] Wang Zhikun, Zhang Xin, Song Yuntong et al. Application of pre-tight disc spring in oil refining unit [J]. Lubrication and Sealing, 2006 (2): 184-185.

[2] Xu Jing, Chen Jing core, Li Hong. Based on knowledge of the disc spring integrated intelligent design [J]. Mechanical Design and Manufacture, 2006 (4): 59-61.

[3] Bouzid A, Nechache A. The modeling of bolted flange joints used with disc springs and tube and tube spacers to reduce relaxation. International Journal of Pressure Vessels and Piping 2010;87:730-6.

[4] Valeev AR, Zotov AN, Kharisov S A. Erratum to Application of Disk Springs for Manufacturing Vibration Isolators with Quasi-Zero Stiffness[J]. Chemical \& Petroleum Engineering, 2016.

[5] Zhang Hui, Yu Wentao, Chen Zhongshuai et al. Research on the development of the hydraulic pulse axial oscillation drag reduction tool [J]. Petroleum Field Machinery, 2014 (7): 73-77.
[6] Zhang Hui. The application of the constant torque tool in the PDC well [J]. Petroleum Machinery, 2015, 43 (12): 15-18.

[7] Liu Yongwang. Design and research of vibration reduction and pressure boosting device in underground [D]. China University of Petroleum, 2007.

[8] Yang Hao. Research and development of [D]. Southwest Petroleum University, 2015.

[9] Yi Xianzhong. Basic characteristic parameters of disk springs analysis [J]. Petroleum Machinery, 1995 (3): 10-17.

[10] Wang Xiaobo. Study on the mechanical properties of the disk spring [D]. Zhengzhou University, 2007

[11] Ozaki S, Tsuda K, Tominaga J. Analyses of the static and dynamic behavior of coned disk springs: Effects of friction boundaries[J]. Thin-Walled Structures, 2012, 59(4):132-143.

[12] Hashiguchi K, Ozaki S. Constitutive equation for friction with the transition from static to kinetic friction and recovery of static friction[J]. International Journal of Plasticity, 2008, 24(11):2102-2124.

[13] Cheng Daxian. Mechanical Design Handbook [M]. Beijing: Chemical Industry Press, 2009:1186-1187.

Volume 6 Issue 12, December 2017 